\title{
THE SECOND OBSTRUCTION FOR PSEUDO-ISOTOPIES
}

\author{
BY ALLEN E. HATCHER ${ }^{1}$
}

\author{
Communicated by Michael Atiyah, May 18, 1972
}

1. Introduction. In this note is announced the completion of the reduction to algebra of the pseudo-isotopy problem for smooth compact manifolds of large dimension. This is to compute $\pi_{0} \mathscr{P}(M, \partial M)$ where, for a smooth manifold $M, \mathscr{P}(M, \partial M)$ is the group of diffeomorphisms of $M \times I$ which are the identity on $M \times\{0\} \cup \partial M \times I, \mathscr{P}(M, \partial M)$ being given the $C^{\infty}$ topology.

THEOREM. If $M$ is compact, connected and of dimension at least seven, then

$$
\pi_{0} \mathscr{P}(M, \partial M) \approx \mathrm{Wh}_{2} \pi_{1} M \oplus \mathrm{Wh}_{1}\left(\pi_{1} M ; Z_{2} \times \pi_{2} M\right) .
$$

For $M$ simply-connected this is Cerf's theorem that $\pi_{0} \mathscr{P}(M, \partial M)$ $=0$ [2]. The $\mathrm{Wh}_{2}$ factor has been described by J. B. Wagoner [7] and, independently, by the author [4]. Partial results on the $\mathrm{Wh}_{1}$ factor have appeared in [3].

We now define and compute $\mathrm{Wh}_{1}\left(\pi_{1} ; Z_{2} \times \pi_{2}\right)$. Let the group $\pi$ act on the abelian group $\Gamma$. Giving $\Gamma$ trivial multiplication, form the group ring $\Gamma[\pi]$. This fits into a split exact sequence

$$
0 \rightarrow \Gamma[\pi] \rightarrow(\Gamma \times Z)_{T}[\pi] \rightarrow Z[\pi] \rightarrow 0,
$$

where the twisting $T$ is given by $\sigma(\alpha \tau)=\alpha^{\sigma} \sigma \tau$ for $\sigma, \tau \in \pi, \alpha \in \Gamma$, and $\alpha^{\sigma}$ denotes the action of $\sigma$ on $\alpha$. Then $\Gamma[\pi]$ is an ideal in $(\Gamma \times Z)_{T}[\pi]$ and the relative group $K_{1} \Gamma[\pi]$ is defined as in [2], [6]. Its elements are represented by matrices $I+A$, where $A$ has entries in $\Gamma[\pi]$.

Proposition. $K_{1} \Gamma[\pi] \approx \Gamma[\pi] /\left(\alpha \sigma-\alpha^{\tau} \tau \sigma \tau^{-1}\right)$, via $[I+A] \mapsto \operatorname{trace}(A)$.

Here '(-)" denotes "additive subgroup generated by -,'.

Define $\mathrm{Wh}_{1}(\pi ; \Gamma)$ as the cokernel of $K_{1} \Gamma[1] \rightarrow K_{1} \Gamma[\pi]$. Note that $\mathrm{Wh}_{1}(\pi ; \Gamma)$ is unrelated to the classical Whitehead group $\mathrm{Wh}_{1} \pi$ since $\Gamma$ was given trivial multiplication, e.g., $\mathrm{Wh}_{1}(\pi ; Z) \neq \mathrm{Wh}_{1} \pi$.

Now let $\pi=\pi_{1}$ and $\Gamma=Z_{2} \times \pi_{2}$, with the usual action of $\pi_{1}$ on $\pi_{2}$ and the trivial action on $Z_{2}\left(Z_{2}=Z / 2 Z\right)$.

AMS 1970 subject classifications. Primary 57D50, 57D80; Secondary 18F25.

${ }^{1}$ Supported by an NSF Postdoctoral Fellowship. 
Corollary. $\mathrm{Wh}_{1}\left(\pi_{1} ; Z_{2} \times \pi_{2}\right) \approx\left(Z_{2} \times \pi_{2}\right)\left[\pi_{1}\right] /\left(\alpha \sigma-\alpha^{\tau} \tau \sigma \tau^{-1}, \beta \cdot 1\right)$ for $\alpha, \beta \in \boldsymbol{Z}_{2} \times \pi_{2}$ and $\sigma, \tau \in \pi_{1}$.

CoRollary. For $M$ as in the Theorem, $\pi_{0} \mathscr{P}(M, \partial M)=0$ if and only if $M$ is simply-connected.

For $\mathrm{Wh}_{1}\left(\pi_{1} ; Z_{2}\right)$ is the direct sum of as many copies of $Z_{2}$ as there are nontrivial conjugacy classes in $\pi_{1}$.

2. Definition of the second obstruction. Computing $\pi_{0} \mathscr{P}(M, \partial M)$ is equivalent to a one-parameter $h$-bordism theorem: Given a path of smooth functions $f_{t}: M \times(I, 0,1) \rightarrow(I, 0,1), 0 \leqq t \leqq 1$, such that

(a) $f_{0}$ and $f_{1}$ have no critical points and

(b) $f_{t} \mid \partial M \times I$ is projection onto $I$,

what is the obstruction to deforming the path $f_{t}$, preserving conditions (a) and (b), so as to eliminate the critical points of all the $f_{t}$ simultaneously?

A generic family $f_{t}$ is most easily described in terms of handlebody theory: At isolated $t$ values there are birth and death points for a pair of mutually cancelling handles of adjacent indices, and in the remaining $t$ intervals the attaching maps of handles change by isotopy. In particular, there are isolated points of "handle addition" where one handle passes over another of the same index.

Assuming from now on that $m=\operatorname{dim} M$ is sufficiently large, it is always possible to push all handles for the one-parameter family into two indices $i$ and $i+1$. The following condition is readily satisfied by introducing extra handle additions if necessary:

$(+)$ At birth or death points, cancelling handle pairs are disjoint from all other handles.

The $\mathrm{Wh}_{2}$ invariant is then defined from the sequence of $i+1 / i$ boundary (or intersection) matrices in $\mathrm{GL}\left(Z\left[\pi_{1}\right]\right)$ as $t$ varies: Each handle addition multiplies this boundary matrix by an elementary matrix $e_{j k}^{\sigma}, \sigma \in \pm \pi_{1}$, and the product $\prod e_{j k}^{\sigma}$ over all handle additions will be a matrix of the form (permutation) $\times$ (diagonal with entries in $\pm \pi_{1}$ ). Such products of elementary matrices, under a suitable equivalence relation (the so-called Steinberg relations [6], and multiplication by terms $e_{p q}^{\tau} e_{q p}^{-\tau} f e_{p q}^{\tau}, \tau \in \pm \pi_{1}$ ), form the group $\mathrm{Wh}_{2} \pi_{1}$, a factor group of $K_{2} Z\left[\pi_{1}\right]$.

The $\mathrm{Wh}_{2}$ invariant vanishes if and only if all handle additions can be eliminated preserving $(+)$. (If $(+)$ is not required to hold one can always eliminate all handle additions.) Then the $i+1 / i$ intersections, i.e., intersections in a level surface of attaching spheres $S_{t}^{i}$ of $(i+1)$-handles with transverse spheres $S_{t}^{m-i}$ of $i$-handles, in a one-parameter family form a one-dimensional compact manifold, with one boundary point at each birth or death point. In order to cancel an $i+1 / i$ handle pair for the 
whole $t$-interval from its birth to its death, the $i+1 / i$ intersection for this pair must consist of exactly one point in each $t$ slice.

There is a Whitney-type procedure for modifying such one-dimensional intersections. The obstruction group is $\left(Z_{2} \times \pi_{2}\right)\left[\pi_{1}\right]$, just as the obstruction group for zero-dimensional intersections is the well-known $Z\left[\pi_{1}\right]$. Considering all $i+1$ and $i$-handles, one obtains a matrix $A$ of "geometric" $i+1 / i$ intersections for the one-parameter family, with entries in $\left(Z_{2} \times \pi_{2}\right)\left[\pi_{1}\right]$. This refines the "algebraic" $i+1 / i$ intersection matrix in $\operatorname{GL}\left(Z\left[\pi_{1}\right]\right)$ which we take to be $I$ in each $t$ slice, as there are no handle additions. The $\mathrm{Wh}_{1}\left(\pi_{1} ; Z_{2} \times \pi_{2}\right)$ invariant of our one-parameter family is represented by the matrix $I+A$.

To show that this $\mathrm{Wh}_{1}$ invariant is well defined we must examine deformations through two-parameter families (these may also be pushed into two indices), and in particular we must define the $\mathrm{Wh}_{1}$ invariant for one-parameter families containing handle additions, where the $i+1 / i$ intersections form only a one-dimensional cell complex $L$ with the handle addition points as vertices. After subdividing $L$ by adding the points $L \cap\{t$ slices containing handle additions $\}$ as vertices, then by a suitable normalization procedure at the vertices of $L$ an invariant in $\left(Z_{2} \times \pi_{2}\right)\left[\pi_{1}\right]$ can be defined for each edge of $L$. Thus in the $j$ th $t$ interval between two successive handle additions, where the algebraic $i+1 / i$ intersection matrix is $M_{j} \in \mathrm{GL}\left(Z\left[\pi_{1}\right]\right)$, there is also a geometric $i+1 / i$ intersection matrix $A_{j}$ over $\left(Z_{2} \times \pi_{2}\right)\left[\pi_{1}\right]$. The $\mathrm{Wh}_{1}$ invariant is then defined as $I+\sum_{j} M_{j}^{-1} A_{j}$.

This definition does not depend on the vanishing of the $\mathrm{Wh}_{2}$ obstruction, so the $\mathrm{Wh}_{1}$ invariant is in fact defined on all of $\pi_{0} \mathscr{P}(M, \partial M)$, which accounts for the direct sum decomposition in the theorem.

3. An application to concordances. Let $e: M^{m} \rightarrow Q^{q}$ be a fixed embedding of smooth compact manifolds. For simplicity assume $\partial M=$ $\partial Q=\varnothing$. By a concordance we mean an embedding $F: M \times I \rightarrow Q \times I$ satisfying $F^{-1}(Q \times\{i\})=M \times\{i\}, i=0,1$, and $F \mid M \times\{0\}=e$. Call $F$ an $h$-concordance if $Q \times I-F(M \times I)$ is an $h$-bordism, or equivalently if $Q \times I-F(\stackrel{\circ}{N} \times I)$ is a relative $h$-bordism, where $N$ is a tubular neighborhood of $M$ in $Q$. Denote by $C(M, Q)\left[C_{h}(M, Q)\right]$ the space of all such [h-] concordances, with the $C^{\infty}$ topology. For the pointed set $\pi_{0} C_{h}(M, Q)$ we have the following:

Proposition. If $q \geqq 7$ there is an exact sequence

$$
\begin{aligned}
\mathrm{Wh}_{2} \pi_{1}(Q-M) & \oplus \mathrm{Wh}_{1}\left(\pi_{1}(Q-M) ; Z_{2} \times \pi_{2}(Q-M)\right) \\
& \stackrel{i_{*}}{\rightarrow} \mathrm{Wh}_{2} \pi_{1} Q \oplus \mathrm{Wh}_{1}\left(\pi_{1} Q ; Z_{2} \times \pi_{2} Q\right) \\
& \stackrel{j}{\rightarrow} \pi_{0} C_{h}(M, Q) \stackrel{k}{\rightarrow} \mathrm{Wh}_{1} \pi_{1}(Q-M) \stackrel{i_{*}}{\rightarrow} \mathrm{Wh}_{1} \pi_{1} Q,
\end{aligned}
$$


where the maps $i_{*}$ are induced by inclusion, $j$ is restriction of a pseudoisotopy on $Q \times I$ to $e(M) \times I \subset Q \times I$, and $k$ is the Whitehead torsion of the h-bordism $Q \times I-F(\stackrel{\circ}{N} \times I)$.

Corollary (Hudson [5]). If $q \geqq 7$ and $q-m \geqq 3$ then $\pi_{0} C(M, Q)=$ $\pi_{0} C_{h}(M, Q)=0$.

By contrast, in codimensions one and two, the $\mathrm{Wh}_{1}\left(\pi_{1} ; Z_{2} \times \pi_{2}\right)$ term provides many nontrivial $h$-concordances, for example when $e$ is the inclusion of a factor $S^{m} \subset S^{m} \times S^{1}$ or, in the relative case, $D^{m} \subset D^{m} \times S^{1}$.

\section{REFERENCES}

1. H. Bass, Algebraic K-theory, Benjamin, New York, 1968. MR 40 \# 2736.

2. J. Cerf, La stratification naturelle des espaces de fonctions différentiables réelles et le théorème de la pseudo-isotopie, Inst. Hautes Ėtudes Sci. Publ. Math. No. 39 (1970), 5-173.

3. A. Chenciner and $\mathrm{F}$. Laudenbach, Contribution à une théorie de Smale à un paramètre dans le cas non simplement connexe, Ann. Sci. Ėcole Norm. Sup. (4) 3 (1970), 109-478.

4. A. Hatcher, $\mathrm{A} \mathrm{K}_{2}$ obstruction for pseudo-isotopies, Ph. D. Thesis, Stanford University, Stanford, Calif., 1971 .

5. J. F. P. Hudson, Piecewise linear topology, University of Chicago Lecture Notes, Benjamin, New York, 1969. MR 40 \#2094.

6. J. Milnor, Introduction to algebraic K-theory, Ann. of Math. Studies, no. 72, Princeton Univ. Press, Princeton, N.J., 1971.

7. J. B. Wagoner, Algebraic invariants for pseudo-isotopies, Proc. Liverpool Singularities Sympos. II, Lecture Notes in Math., no. 209, Springer-Verlag, Berlin and New York, 1971.

Department of Mathematics, Princeton University, Princeton, New Jersey 08540 